\title{
HISTORIA DE LOS PROGRAMAS DE FORMACIÓN Y TITULACIÓN DE MÉDICOS ESPECIALISTAS EN EL PERÚ
}

\author{
Zuño Burstein Alva1,a,b,c
}

\begin{abstract}
RESUMEN
En el presente artículo se hace referencia histórica a las disposiciones legales de reconocimiento de la formación y titulación de los médicos especialistas en el Perú mediante programas de estudios universitarios, previstos desde el año 1928, que culminaron el año 1973, con la autorización legal de la autoridad pertinente del Estado, de implementar el Programa de Segunda Especialización en Medicina Humana y a otorgar el título de especialista a nombre de la Nación al término de un programa universitario de residentado y capacitación especializada mediante la "modalidad escolarizada" y el reconocimiento universitario de la "modalidad no escolarizada". En el Perú quedó establecido hasta la actualidad, que los títulos profesionales de especialista en medicina humana a nombre de la nación, tanto en la "modalidad escolarizada" como en la "no escolarizada" solo pueden ser otorgados por las universidades nacionales o privadas autorizadas para esta misión y que, además, ninguna otra institución pública o privada puede arrogarse esta función que corresponde exclusivamente a la universidad peruana.
\end{abstract}

Palabras clave: Especialización; Educación médica; Perú (fuente DeCS BIREME).

\section{HISTORY OF TRAINING AND CERTIFICATION PROGRAMS OF MEDICAL SPECIALISTS IN PERU}

\begin{abstract}
In this paper, historical reference is made about legal provisions for recognition of the training and certification of medical specialists in Peru through university programs provided since 1928 and culminating in 1973 with the legal authorization by the relevant state authority to implement the Second Specialization Program in Human Medicine and to grant the Certification of Specialist in the Name of the Nation upon completion of a university residency program and specialized training by the "regular modality", and the recognition by the university with the "non-regular modality". In Peru it has been established to the present that the Professional Certifications of Specialists in Human Medicine "in the Name of the Nation", both in "regular modality" as well as in "non-regular modality", can only be granted by public or private universities authorized for this mission and, besides, no other public or private institution can claim this role that corresponds exclusively for the Peruvian University.
\end{abstract}

Key words: Specialization; Education, medical; Peru (source: MeSH, NLM).

Los estudios para obtener el título universitario de especialista son considerados desde el año 1928 en el Estatuto Universitario promulgado por la Ley 6041, esta prerrogativa solo se otorgó a las facultades de Medicina y de Derecho de la Universidad Nacional Mayor de San Marcos de Lima (UNMSM). Esta disposición se mantiene en las siguientes leyes y estatutos universitarios, pero desaparece en el texto de la Ley 13417 del año 1963. Por esta razón, la Facultad de Medicina de "San Fernando" de la UNMSM se apresura a crear su Escuela de Graduados, en el que estableció el sistema de residencias para formar especialistas en las distintas ramas de la actividad médica, pero no otorgaba el título de especialista, sino un diploma en el que se consignaba que un médico-cirujano: «Ha cumplido satisfactoriamente con el Programa de Residencia en la especialidad de...», con fechas de inicio y término de los cursos y actividades programadas. Este diploma lo firmaba el decano de la Facultad de Medicina y el director de la Escuela de Graduados ${ }^{(1)}$.

El Estatuto General de la Universidad Peruana, promulgado por Decreto Ley 17437 el año 1972, establece que los programas de posgrado (cursos de actualización y/o refresco) y los programas de residencias

\footnotetext{
Universidad Nacional Mayor de San Marcos. Lima, Perú

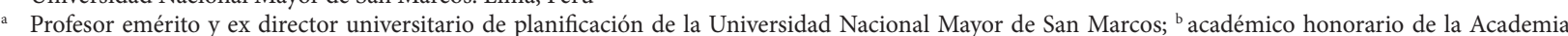
Nacional de Medicina; ${ }^{\mathrm{C}}$ exmiembro del Comité de Vigilancia Ética y Deontología del Colegio Médico del Perú.

Recibido: 09-07-14 Aprobado: 20-08-14
}

Citar como: Burstein Alva Z. Historia de los programas de formación y titulación de médicos especialistas en el Perú. Rev Peru Med Exp Salud Publica. 2014;31(3):598-600. 
se ubiquen en el ciclo de segunda especialización de la Dirección de Programas Académicos de Medicina Humana. La primera especialización termina con la obtención del título de médico cirujano.

Por Resolución 1226-73-CONUP del 18 de enero de 1973, el Consejo Nacional de la Universidad Peruana (CONUP) autorizó a la UNMSM a implementar su Programa de Segunda Especialización en Medicina Humana y a otorgar el título de especialista, que por primera vez se otorgaría en el Perú a nombre de la Nación, al terminar un riguroso programa de residentado y capacitación especializada, siguiendo la "modalidad escolarizada".

Antes de la existencia de estos programas, los profesionales médicos se hacían especialistas mediante el proceso de autoeducación, interaprendizaje o estudios realizados en forma independiente bajo la modalidad no escolarizada.

El CONUP, por Resolución 1556-74-CONUP, del 6 de febrero de 1974, consideró que era conveniente otorgar el título de especialista mediante la "modalidad no escolarizada" para así regularizar la situación de muchos profesionales, además, ninguna otra institución pública o privada podía arrogarse esta función, que corresponde exclusivamente a la universidad peruana. El CONUP, amparado por el Art. 152 del Decreto Ley 19326, resuelve autorizar al Programa de Segunda Especialización en Medicina Humana de la Universidad Nacional Mayor de San Marcos para otorgar el título de "médico cirujano especialista en..." a nombre de la Nación Peruana en la "modalidad no escolarizada".

El 16 de octubre de 1964, por Ley 15173, coordinada con el Decreto Ley 17239 del 29 de noviembre de 1968, se crea el Colegio Médico del Perú (CMP); la Comisión Redactora de los Estatutos y Reglamentos, lo conformaron, como representantes de los programas académicos de Medicina Humana, los Dres. Elmer Alegría y Zuño Burstein. Constituido estructuralmente el CMPy, en cumplimiento de sus fines, se creó el registro de matrícula de los médicos cirujanos, el de los especialistas y el de las sociedades médico-científicas, asimismo, se crearon los comités calificadores competentes para cada objeto. Pero poco tiempo después de creado el CMP comenzó a otorgar, en ceremonias públicas, títulos de especialista por la modalidad no escolarizada a médicos cirujanos calificados por él.

El 22 de noviembre de 1972 el Consejo Ejecutivo de la UNMSM designó una comisión compuesta por el Dr. Zuño Burstein (Director Universitario de Planificación), el Dr. Andrés Rota (Director de Programas Académicos de Medicina Humana) y el Dr. Elmer Alegría (en representación de la Escuela de Graduados de Medicina de la UNMSM) para sustentar ante el CONUP la suspensión de la atribución que, ilegalmente, estaba asumiendo el CMP al otorgar títulos de especialización a nombre de la Nación y, además, para proponer soluciones a los problemas del Programa de Segunda Especialización en Medicina Humana de la UNMSM.

El problema creado se resolvió, y quedó establecido, hasta la actualidad, que los títulos profesionales de especialista, tanto en la modalidad escolarizada como en la modalidad no escolarizada, solo podían ser otorgados por las universidades nacionales y privadas que fueran autorizadas para esa misión, una vez aprobados sus programas y reglamentos, después de una exhaustiva evaluación por la autoridad competente (CONUP en aquel entonces). EI CMP quedó con la atribución legal de registrar automáticamente los títulos de especialista otorgados por las universidades, autorizando su ejercicio profesional y, por un tiempo limitado, autorizar el ejercicio profesional bajo condiciones especiales, previa calificación, de quienes no lo tuvieran y vigilando en ambos casos, como le corresponde, el cumplimiento de la ética y deontología médica.

En la UNMSM el Programa de Segunda Especialización (2) comprende, desde su creación en el año 1973 (Resolución Rectoral 38145 UNMSM y Res. CONUP 1226-73), la enseñanza y las actividades dentro de las ciencias básicas y clínicas. Su currículo abarca fundamentalmente prácticas profesionales intensivas, asignaturas del más alto nivel y de actualidad, actividades no cognoscitivas y actividades de consejería y orientación. Su duración no debe ser menor de seis semestres con un total de 120 créditos. Para matricularse es requisito indispensable poseer el título profesional de médico cirujano y el ingreso es por estricto concurso de méritos.

En cuanto a los ambientes y equipos que utiliza el Programa de Segunda Especialización de la UNMSM, hay que mencionar que, con fecha 13 de abril de 1971, se expidió el Decreto Supremo 0055-71SA, por el que se dispone que los centros asistenciales del sector salud y trabajo presten facilidades para la docencia médica universitaria; se estableció que el residentado de especialización se efectuaría en los centros asistenciales acreditados, previa evaluación. Las instituciones de salud estatales y paraestatales que solicitan la preparación de los médicos especialistas que necesitan, tienen que crear las plazas, encargándose de la cobertura económica y deben coordinar con la universidad la convocatoria a concurso, ajustándose a lo estipulado en el Reglamento Universitario de 


\section{Programas de Segunda Especialización de Medicina} Humana, autorizado por Resolución del CONUP 155674 CONUP del 6 de febrero de 1974.

El 15 de marzo de 1974, la Dirección de los Programas Académicos de Medicina Humana de la UNMSM aprueba 27 comités de especialización en medicina humana ${ }^{(5)}$, entre los que se encontraba el Comité de Dermatología (2), presidido en su inicio por el Prof. Dr. Aizic Cotlear e integrado por los Drs. Dante Mendoza, José San Martin y Alejandro Morales y el de Medicina Tropical presidido por el Prof. Dr. Zuño Burstein e integrado por los Drs. Wilfredo Gardini, José Neyra, César Náquira y Abelardo Tejada. Desde aquel entonces se regularizaron progresivamente todos los programas de especialización existentes en la actualidad en la UNMSM, así como en todas las universidades del país con programas académicos o facultades de Medicina Humana.

El 3 de Marzo de 1974 y el 16 de Abril de 1975, la UNMSM ${ }^{(6,7)}$ a través de su Programa de Segunda Especialización en Medicina Humana, publica en los diarios de circulación nacional las disposiciones, trámites y requisitos para la obtención de los títulos de especialista en Medicina Humana por la modalidad no escolarizada (4) para 28 especialidades y las normas para la formación de especialistas. El 22 de abril de 1974 la UNMSM, asumiendo públicamente sus derechos y obligaciones establecidos por ley y en vías de regularización, hace entrega de los títulos de especialista en las diversas ramas de la medicina mediante la "modalidad escolarizada". Esta entrega de títulos a los que habían culminado satisfactoriamente su entrenamiento en la ex-Escuela de Graduados se realizó en sesión pública solemne en el Palacio Municipal de Lima, por invitación del rector de la UNMSM y del presidente del CONUP, se contó con la presencia del Ministro de Educación y del alcalde del Concejo Provincial de Lima. Con este acto se hizo pública reafirmación de que en el Perú el único organismo autorizado para otorgar títulos profesionales de especialista a nombre de la Nación era la universidad peruana.

\section{REFERENCIAS BIBLIOGRÁFICAS}

1. Burstein Z. Historia de la Dermatología en el Perú: historia de la formalización legal de la especialidad en el Perú. En: Galimberti R, Pierini AM, Cervini AB. Historia de la dermatología latinoamericana. París: Privat; 2007. p. 337-43.

2. Universidad Nacional Mayor de San Marcos, Facultad de Medicina. Disposiciones legales y organización del Programa de Segunda Especialización. Lima: UNMSM; 1974.

3. Universidad Nacional Mayor de San Marcos, Facultad de Medicina. Programa de Segunda Especialización en Medicina Humana. Comités de
Especialidad del Programa de Segunda Especialización en Medicina Humana. Lima: UNMSM; 1974.

4. Burstein Z. Desarrollo y avances de la Dermatología en el Perú durante los últimos 30 Años. Diagnóstico. 2009;48(3):118-25.

5. Universidad Nacional Mayor de San Marcos. Programa de Segunda Especialización en Medicina Humana. Título de Especialista. El Comercio, 3 marzo 1974.

6. Universidad Nacional Mayor de San Marcos. Programa de Segunda Especialización en Medicina Humana. Títulos de Especialista en
Medicina Humana. El Comercio, 16 abril 1975.

7. Universidad Nacional Mayor de San Marcos, Facultad de Medicina. Normas y procedimientos para la obtención del título de especialista por la modalidad escolarizada y no escolarizada. Lima, Perú: UNMSM, 1974.

Correspondencia: Zuño Burstein

Dirección: Capac Yupanqui 1400, Jesús

María, Lima

Teléfono: (511) 471-9920 anexo: 122

Correo electrónico: zburstein@ins.gob.pe 